\title{
Board 88: Support Remote Collaboration in Virtual Computer Labs
}

\author{
Prof. Xiaolin Hu, Georgia State University
}

Dr. Xiaolin Hu is an Associate Professor in the Computer Science Department and Director of the Systems Integrated Modeling and Simulation (SIMS) Lab at Georgia State University, USA. He received his $\mathrm{Ph} . \mathrm{D}$. degree from the University of Arizona in 2004. His research interests include modeling and simulation theory and application, complex systems science, agent and multi-agent systems, and advanced computing in parallel and cloud environments. His work covers both fundamental research and applications of computer modeling and simulation. He was a National Science Foundation (NSF) CAREER Award recipient

\section{Mr. Hai Le, Georgia State University}

Hai Le, a native of Vietnam, is a PhD student in the Computer science at Georgia State University at Atlanta. He is one of the members of collaborative virtual computer lab developing team. His current research focuses on Agent-Based simulation and modeling, particularly on emergent behaviors. His future goals include working as a professor and focus on Simulation and Modeling research topics.

\section{Dr. Yuan Long, Georgia State University}

YUAN LONG is affiliated with Georgia State University. Her research interests include machine learning, big data analysis, and high-performance computing. Her email address is ylong4@gsu.edu.

\section{Anu G. Bourgeois, Georgia State University}

Anu G. Bourgeois is an Associate Professor in the Department of Computer Science at Georgia State University. She received her Masters and Ph.D. in Electrical and Computer Engineering from Louisiana State University in 1997 and 2000, respectively. Her research interests include parallel and distributed computing, wireless networks, security and privacy, fault tolerant computing, and STEM education. She is a senior member of the IEEE.

\section{Prof. Yi Pan, Georgia State University}

Dr. Yi Pan is a Distinguished University Professor of the Department of Computer Science and Associate Dean of Arts and Sciences at Georgia State University. He is also a visiting Changjiang Chair Professor at Central South University in China. Dr. Pan received his B.Eng. and M.Eng. degrees in computer engineering from Tsinghua University, China, in 1982 and 1984, respectively, and his Ph.D. degree in computer science from the University of Pittsburgh, USA, in 1991.

Dr. Pan's research interests include parallel and cloud computing, wireless networks, and bioinformatics. Dr. Pan has published more than 330 papers including over 150 SCI journal papers and 50 IEEE Transactions papers. In addition, he has edited/authored 40 books. He has received many awards from organizations such as IEEE, NSF, AFOSR, JSPS, IBM, ISIBM, IISF and Mellon Foundation. Dr. Pan has served as an editor-in-chief or editorial board member for 15 journals including 7 IEEE Transactions and a guest editor for 12 special issues for 10 journals including 2 IEEE Transactions. He has organized numerous international conferences and workshops and has delivered over 40 keynote speeches at international conferences around the world.

Dr. Pan is a "Great Master Face-to-Face" Series Speaker (2012), an IEEE Distinguished Speaker (20002002), a Yamacraw Distinguished Speaker (2002), a Shell Oil Colloquium Speaker (2002), and a senior member of IEEE. He is listed in Men of Achievement, Who's Who in Midwest, Who's Who in America, Who's Who in American Education, Who's Who in Computational Science and Engineering, and Who's Who of Asian Americans. 


\section{Support Remote Collaboration in Virtual Computer Labs}

\section{Introduction}

Computer labs are commonly used in computing education to help students reinforce the knowledge obtained in classrooms and to gain hands-on experience on specific learning subjects. While traditional computer labs are based on physical computer centers on campus, more and more virtual computer lab systems (see, e.g., [1, 2, 3, 4]) have been developed that allow students to carry out labs on virtualized resources remotely through the Internet. Virtual computer labs make it possible for students to use their own computers at home, instead of relying on computer centers on campus to work on lab assignments. However, they also make it difficult for students to collaborate, due to the fact that students work remotely and there is a lack of support of sharing and collaboration. This is in contrast to traditional computer labs where students naturally feel the presence of their peers in a physical lab room and can easily work together and help each other if needed.

Funded by NSF's Division of Undergraduate Education, this project develops a collaborative virtual computer lab (CVCL) environment to support collaborative learning in virtual computer labs. The CVCL environment leverages existing open source collaboration tools and desktop sharing technologies and adds new functions unique to virtual computer labs to make it easy for students to collaborate while working on computer labs remotely. It also implements several collaborative lab models to support different forms of collaboration in both formal and informal settings. We have developed the main functions of the CVCL environment and begun to use it in classes in the Computer Science (CS) department at Georgia State University.

While the original project focuses on computer labs in its traditional sense, the issue of lack of collaboration applies to much broader learning settings where students work on tasks or assignments on computers, with or without being associated with a lab environment. Due to the high mobility of students in modern campuses and the fact that many learning activities are carried out over the Internet, computer-based learning increasingly happen in students' personal spaces (e.g., homes, apartments), as opposed to public learning spaces (e.g., laboratories, libraries). In these personal spaces, it is difficult for students to get help from classmates or teaching assistants (TAs) when encountering problems. As a result, collaborative learning is difficult and rare. This is especially true for urban universities such as Georgia State University where a significant portion of students are part-time students and/or commute. To address this issue, we intend to broaden the concept of "virtual computer lab" to include general computerbased learning happening in "virtual space," which is any location where people can meet using networked digital devices [5]. Virtual space is recognized as an increasingly important part of "learning spaces" and asks for support from both the technology aspect and learning theory aspect [5]. Collaborative learning environments that support remote collaboration in virtual computer labs would fill an important need in this broader trend.

\section{Overview of the CVCL Environment}


To support remote collaboration in virtual computer labs, we have developed an integrated collaborative virtual computer lab (CVCL) environment [6]. The CVCL environment follows a layered architecture to run on top of existing virtual computer lab systems. This means it can work with different virtual computer lab systems. The CVCL environment supports several functions that are essential for collaborative virtual computer labs: 1) lab and user management, 2) real time communication, 3) remote desktop sharing. The lab and user management deals with lab creation, session management, and user data to ensure students join the right labs at the right time. The real time communication allows students of the same lab to communicate with each other through chat, voice call, and video call. The remote desktop sharing allows a student to share his/her desktop with other participants of the same lab. This function is especially useful in virtual computer labs so that multiple students can see the same desktop while working together (e.g., debugging a computer program). To achieve these functions, we leverage existing open source web conferencing and desktop sharing tools and integrate them into the CVCL environment. We also implemented several collaborative lab models to support different forms of collaboration in both formal and informal settings, including 1) shared remote collaboration, where multiple students share and work on the same desktop (similar to the software development paradigm pair programming [7]); 2) virtual study room, where students work on their own computers but can communicate with others when needed; and 3) virtual tutoring center, where students can have real-time one-to-one interaction with tutors. More details about these collaborative lab models, as well as the CVCL environment, can be found in [6].

\section{Implementing CVCL in CS Courses}

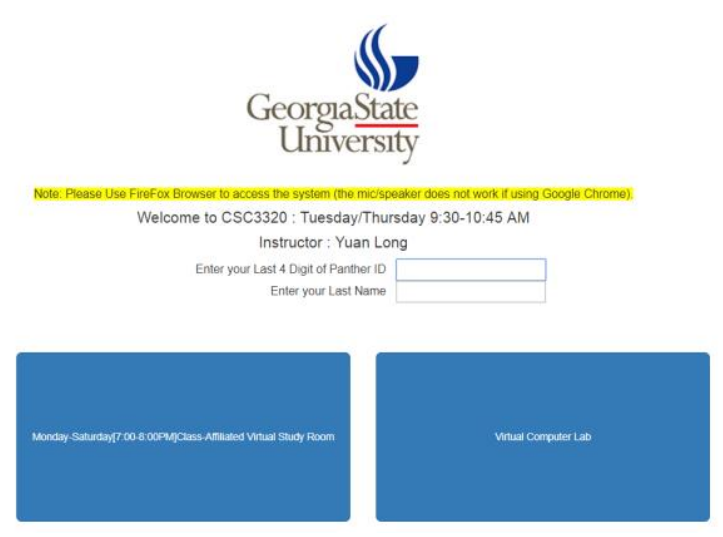

(a) login web portal

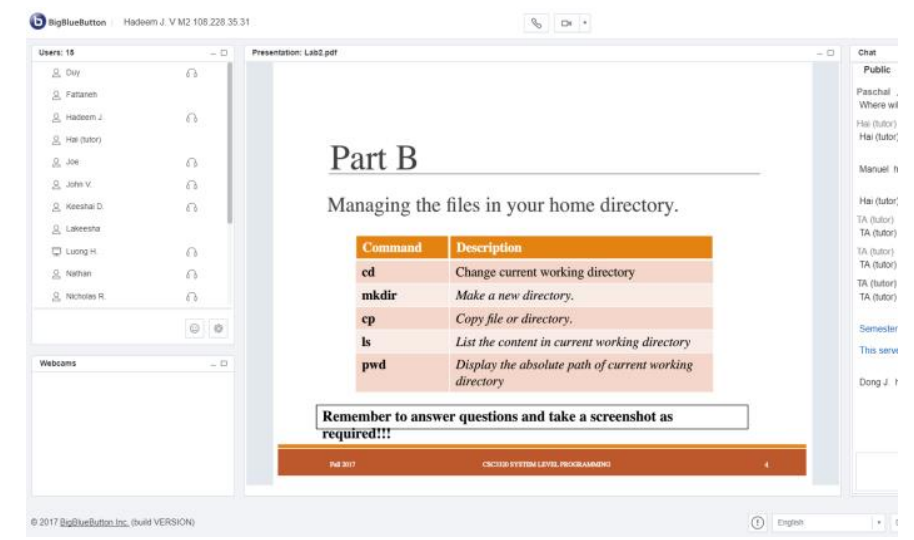

(b) class-affiliated virtual study room

Figure 1: Implementing CVCL in the System Level Programming course

To evaluate the developed environment, we incorporated it in three classes of the System Level Programming course of the CS department in the 2018 fall semester. This course requires students to practice Unix commands and C programming in a Unix/Linux environment. Based on the three collaborative lab models described above, we identified multiple ways of using the CVCL environment to support computer labs associated with this course. These include: 1) class-affiliated virtual study room that offers a common virtual space for students of a class to discuss and propose solutions for practice problems posted by the instructor; 2) virtual tutoring center where students can have online meetings with TAs of the class in designated tutoring hours (similar to what happens in a physical tutoring center or office hours on campus); 3) virtual computer lab where students carry out computer labs under the guidance of a lab assistant; 
4) ad-hoc virtual study room where students reserve virtual study rooms and invite classmates/friends to study together whenever they want to. Figure 1(a) shows a snapshot of the login web portal for one of the classes, where students can login to the class-affiliated virtual study room and virtual computer lab. Figure 1(b) shows a snapshot of the class-affiliated virtual study room after login. The top left window shows all the students (and TAs) who are in the study room; the central window shows the presentation slides (or other documents) that the instructor shares with the class; the chat window on the right (only a portion of it is shown in the figure) allows students to chat with each other.

The CVCL environment was introduced to the students at around the middle of the 2018 fall semester. Our goal was to have an initial test of the environment in real classes before carrying out full-scale evaluation. We demonstrated the CVCL environment in the classes and encouraged students to use the class-affiliated virtual study room and the virtual tutoring center (tutoring hours were Monday-Friday 7-8pm). The virtual computer lab model was not used. Students used the environment voluntarily - they were not required to use it as part of the course. Comprehensive evaluation data were not collected in this initial test. Nevertheless, students were asked to voluntarily complete a survey at the end of the semester. Appendix A shows the survey questions that were asked. For most of these questions, students needed to circle one answer from a list of choices. The majority of the questions in this survey focused on background information related to students' perceptions about collaborative virtual computer labs. Out of all three classes that had 160 students, 70 students responded to the survey. Below we show the results for four of the survey questions that are closely related to this paper.

Figure 2 shows the survey results for Question 1 and Question 2, which asked about students' general view of collaborative computer labs and the places that they typically work on programming/homework assignments. As can be seen from Question 1, 72.8\% students thought collaborative computer lab would be useful for their study in CS courses (Strongly agree or Agree). From Question 2, it can be seen that the majority of students (75.7\%) typically work alone at home when doing their programming/homework assignments. This is consistent with the broader trend that many learning activities happen outside the campus (as described in Section 1). This result signifies the need for tools and learning environments to support remote collaboration in virtual computer labs.

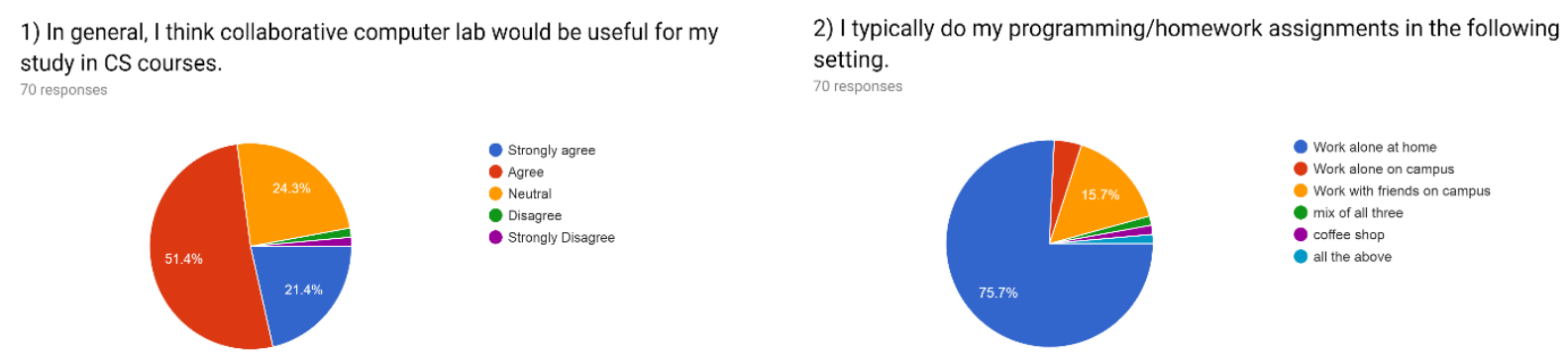

Figure 2: Survey results for Question 1 and Question 2

Figure 3 shows the survey results for Question 12 and Question 13, which collected students' opinions about the usefulness of the CVCL environment. Due to the limited time and degree of integrating the environment into the classes, many students did not have much experience with the environment. We suspect the results for these two questions could be different if students had 
more time using the system. Nevertheless, it can be seen that most students thought the functions of the CVCL environment were useful. Specifically, $56.1 \%$ of the students thought the classaffiliated virtual study room was very useful or somehow useful; $64.6 \%$ of the students thought the virtual tutoring center was very useful or somehow useful.

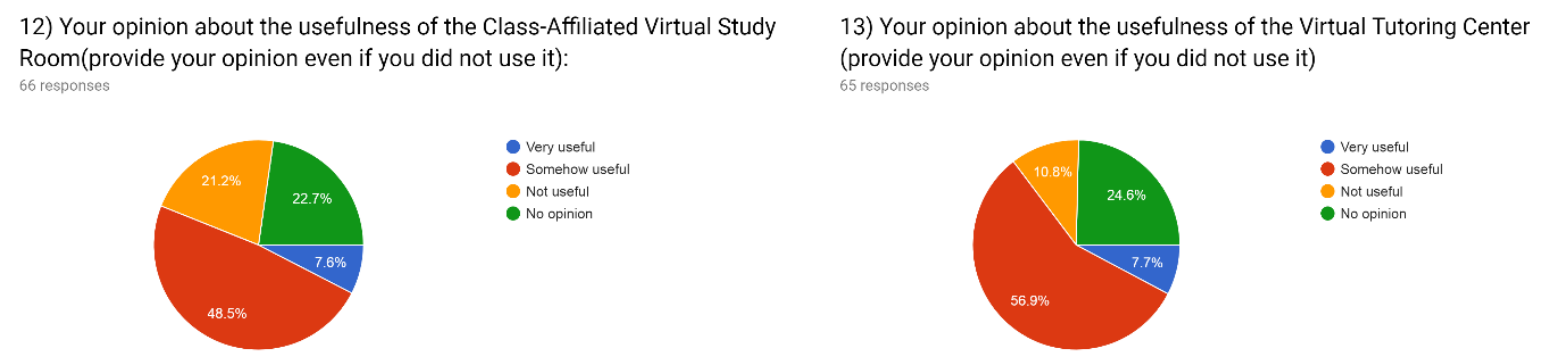

Figure 3: Survey results for Question 12 and Question 13

\section{Conclusion and Future Work}

Computer-based learning is increasingly happening in virtual spaces over the Internet. To address difficulty of collaborative learning in virtual spaces, we developed a collaborative virtual computer lab environment that supports remote collaboration in virtual computer labs. The developed environment was incorporated in three sections of a CS class, reaching a total of 160 students. Preliminary survey results showed that students generally have a need of collaborative learning in virtual computer labs and also view the different functions of the CVCL environment to be useful. Several issues were also discovered, including how to attract students to initially use the system and how to make the system more stable and user friendly. Future work includes addressing these and other issues to improve the system, carrying out more comprehensive tests and evaluation of the environment in more courses.

\section{Acknowledgement}

This work was supported by the National Science Foundation IUSE program under Grant \#1712384: Collaborative Learning in Cloud-based Virtual Computer Labs. Any opinions expressed in this paper are those of the authors and are not that of the National Science Foundation.

\section{References}

1. L. Xu, D. Huang, and W. T. Tsai, "V-Lab: A Cloud-based Virtual Laboratory Platform for Hands-on Networking Courses," Proc. 17th Annu. ACM ITiCSE, pp. 256-261, 2012.

2. L. Xu, D. Huang and W. T. Tsai, "Cloud-Based Virtual Laboratory for Network Security Education," IEEE Transactions on Education, vol. 57, no. 3, pp. 145-150, Aug. 2014.

3. J. Son, C. Irrechukwu, and P. Fitzgibbons, "Virtual lab for online cyber security education," Communication of the IIMA, vol. 12, no. 4, art. 5, 2012.

4. "Apache VCL.", 2012 [Online] Available: https://vcl.apache.org.

5. Malcolm Brown, Book chapter: "Learning Spaces," in: Oblinger, D., and Oblinger, J., editors, Educating the Net Generation, an Educause eBook, 2005, chapter 12. 
6. X. Hu, H. Le, A. Bourgeois, and Y. Pan, "Collaborative Learning in Cloud-based Virtual Computer Labs," Proc. Frontiers in Education 2018 (FIE 2018), 2018

7. W. Laurie and R. Kessler, Pair Programming Illuminated. Addison-Wesley, 2002.

\section{Appendix A: Survey Questions}

1. In general, I think collaborative computer lab would be useful for my study in CS courses. [circle one]

2. I typically do my programming/homework assignments in the following setting. [circle one]

3. In general, I have difficulty in getting help from others (friends or instructors) when I encounter problems in programming/homework assignments. [circle one]

4. In the past, my frequency of using collaboration tools (e.g., video/voice conference, chatting tools, phone calls, desktop sharing tools) in programming/homework assignments is: [circle one]

5. When I use collaboration tools in my programming/homework assignments, the most used function is: [circle one]

6. Provide up to three collaboration tools (software systems or Apps) that you have used:

7. I have difficulty in finishing the programming/homework assignments of this course (CSC3320: System Level Programming) by myself. [circle one]

8. In this semester, how many times did you go to the tutoring center (Sports Arena, Room 107) or the instructor/TAs' office hours related to this course? [circle one]

9. If you never or rarely used the tutoring center or office hours, the reason was: [circle one]

10. How many times did you login to the CVCL system that the instructor has introduced in this class? [circle one]

11. If you never or rarely used the CVCL system, the reason was: [circle one]

12. Your opinion about the usefulness of the Class-Affiliated Virtual Study Room (provide your opinion even if you did not use it): [circle one]

13. Your opinion about the usefulness of the Virtual Tutoring Center (provide your opinion even if you did not use it): [circle one]

14. Your evaluation of the usability and functionality of the CVCL system. [circle one]

15. Please add any additional comments about the CVCL system, either what you liked or did not like, and make any suggestions for improvement here. 